\title{
Dishevelled-2 modulates osteogenic differentiation of human synovial fibroblasts in osteoarthritis
}

\author{
LIHUA ZHANG, LUAN LUAN and YINGYING MA \\ Department of Rheumatology, Jining No. 1 People's Hospital, Jining, Shandong 272000, P.R. China
}

Received December 12, 2017; Accepted March 29, 2018

DOI: $10.3892 / \mathrm{mmr} .2018 .8975$

\begin{abstract}
Dishevelled (Dvl)-2 represents one of the cytoplasmic proteins, which serves as a pivotal hub in signaling intermediates through a number of different signaling pathways associated with the Wnt family. The aim of the present study was to investigate the roles and mechanisms of Dvl-2 on synovial fibroblasts (SFBs) in osteoarthritis (OA). A Cell Counting kit-8 (CCK-8) assay was used to determine cell viability. An alkaline phosphatase (ALP) test kit was used to measure the activity of ALP. Western blot and reverse transcription-quantitative polymerase chain reaction analysis were used to evaluate the protein and mRNA expression, respectively. The results suggest that depletion of Dvl-2 significantly decreased the expression of osteoprotegerin (OPG) and ALP $(\mathrm{P}<0.05)$ and significantly increased the expression of receptor activator of nuclear factor- $\kappa \mathrm{B}$ ligand (RANKL), ALP, osteonectin $(\mathrm{ON})$, osteocalcin $(\mathrm{OCN})$ and osterix $(\mathrm{P}<0.05)$. In addition, the depletion of Dvl-2 also significantly inhibited the expression of runt-related transcription factor 2 (Runx-2) and $\beta$-catenin in SFBs $(\mathrm{P}<0.05)$. The effect of Dvl-2 over-expression was opposite to the effect of Dvl-2 silencing. The inactivation of Wnt3a reversed the effect of Dvl-2 silencing. In conclusion, the results indicate that Dvl-2 regulated osteogenic differentiation of SFBs in OA.
\end{abstract}

\section{Introduction}

Osteoarthritis (OA) also known as degenerative arthritis or proliferative arthritis is a joint disease characterized by the cartilage progressive destruction, which results from the excessive degradation of cartilage extracellular matrix components (1). The risk factors of OA is various, which can be classified as person-level factors, such as sex, age, race, genetics, and diet,

Correspondence to: Dr Yingying Ma, Department of Rheumatology, Jining No. 1 People's Hospital, 6 Jiankang Road, Fuqiao Street, Jining, Shandong 272000, P.R. China

E-mail: yingyingma25my@163.com

Key words: dishevelled-2, osteoarthritis, ossification, synovial fibroblasts, Wnt3a, osteoprotegerin, receptor activator of nuclear factor- $\kappa \mathrm{B}$ ligand, alkaline phosphatase and joint-level factors involving malalignment, injury, and abnormal loading of the joints (2). Though OA leads to great suffering to patients, seriously affects their life qualities, and further raises a serious burden on the entire social economy. However, there is no drug that is able to effectively delay or prevent the progression of OA. Joint replacement is the only medical treatment during the middle and late stages of OA diease. However, studies have shown that synovial inflammation plays a crucial role in the development and progression of OA. Due to the high expression of inflammatory mediators in early OA synovial tissues, acute synovitis may be the origin of OA (3). Synovial membrane is a special type of cementitious tissue that consists of a lining layer, a lining under-layer, and an outer edge of the lining fuses with the joint capsule (4). Synovial cells can be divided into macrophages, fibroblasts, and dendritic cells. In addition, synovial fibroblasts (SFBs) can secrete collagen, fibronectin, osteonectin (ON), and hyaluronic acid (5). It has also been reported that SFBs can generate multiple cytokines, involving osteoprotegerin (OPG) and receptor activator of nuclear factor- $\kappa \mathrm{B}$ ligand (RANKL) $(6,7)$. Hence, the SFBs from joints may become the key in OA joint treatment.

As a prevalent membrane-bound glycoprotein, alkaline phosphatase (ALP) promotes the hydrolysis of phosphate monoesters at basic $\mathrm{pH}$ values (8). ALP is expressed in several osteocytes, including osteoblasts, osteoclasts, and bone marrow stromal cells (9-11). Studies have found that the activity of ALP was closely associated with the bone formation (12), mineral bone disorder (13), and osteogenic differentiation (14). Although ALP is expressed in many mammalian tissues and has been studied for several years, it is still little known. Moreover, regulatory mechanisms of ALP in the ossification of SFBs in OA are still little known to us.

A large number of co-receptors, receptors, ligands, and regulatory components are involved in the complex Wnt pathway (15) that proved to participate in several evident signaling events, such as $\beta$-catenin signaling activation (16). Researchers found that Wnt pathway modulates the maturation, differentiation as well as the apoptosis of osteoblasts and osteoclasts, therefore maintaining the balance of organism's bone metabolism (17). There are usually two types of Wnt pathways, which are the classical $\beta$-catenin-dependent pathway and non-classical $\beta$-catenin-independent pathway (18). In the classical $\beta$-catenin-dependent Wnt pathway, dishevelled acts as a key molecule that upregulates the Wnt pathway (19). A recent 
research indicates that the Wnt pathway may serve as a target for OA therapy (20). SFBs, as one of the bone progenitor cells, have a strong ability of reproduction division in vitro in organizational engineering. Moreover, FB could be directly turned into bone cells without $\mathrm{OB}$ cells, and which was recognized as a most effective way in bone formation (21). Therefore, it was important to further explore the exact mechanisms of Wnt pathway in osteogenic differentiation of SFBs.

Dishevelled (Dvl) is one of the cytoplasmic proteins, and it servea as a pivotal hub in signaling intermediates through a number of different signaling pathways of Wnt family (22). Three dishevelled homologs-Dvl-1/2/3 are expressed in human and mice. Dvl-2 has effective impacts on the progressions of gliomas, prostate tumor and esophageal squamous cell carcinoma (23-25). Due to the osculating relationship between Dvl and Wnt pathway (19), we thereby set out to investigate the definite roles and mechanisms of Dvl-2 in the ossification of SFBs in OA via regulating the Wnt pathway.

In the current study, we analyzed the correlation between Dvl-2 and the ossification of SFBs in OA. Furthermore, it was also fascinating to investigate the exact role and mechanisms of Dvl-2 together with Wnt pathway in the ossification of SFBs in OA.

\section{Materials and methods}

Cell culture, genes, plasmids, and inhibitor. Human SFBs were obtained as previously described (26). Synovial membranes were obtained from 16 OA patients (mean age, $65 \pm 4.5$ years) during arthroplastic surgery with the informed consent from patient and the approval of Ethics Committee of Jining No. 1 People's Hospital (Jining, China). The synovial membranes collected from end-stage joint narrowed space of hip and knee joints. The dissected tissues were incubated in DMEM (Gibco; Thermo Fisher Scientific, Inc., Waltham, MA, USA) containing $1 \mathrm{mg} / \mathrm{ml}$ collagenase (Wako Pure Chemical Industries, Ltd., Osaka, Japan) with shaking for $90 \mathrm{~min}$ at $37^{\circ} \mathrm{C}$. The cells were centrifuged at $400 \mathrm{x} \mathrm{g}$ at $20^{\circ} \mathrm{C}$ for $30 \mathrm{~min}$. Then, released SFBs were maintained in tissue culture flasks at $37^{\circ} \mathrm{C}$ for $1 \mathrm{~h}$. Then, SFBs were incubated in DMEM supplemented with $10 \%$ heat inactivated FBS (Gibco; Thermo Fisher Scientific, Inc.), $10 \%$ penicillin/streptomycin in a $5 \% \mathrm{CO}_{2}$ atmosphere at $37^{\circ} \mathrm{C}$. Dvl-2 RNA and Dvl-2 siRNA were respectively cloned into 2 pcDNA3.1(+) empty vectors-Dvl-2 (Invitrogen; Thermo Fisher Scientific, Inc.) and si-Dvl-2. IWR-1-endo (Beyotime, Shanghai, China) was used as a Wnt inhibitor.

Grouping. Control group (SFBs), NC group (SFBs transfected with empty vector), Dvl-2 group (SFBs transfected with Dvl-2), and si-Dvl-2 group (SFBs transfected with si-Dvl-2) were prepared as four treatment groups in this study. At least three independent experiments were performed.

Cell viability analysis. Cell Counting Kit-8 (CCK-8; Beyotime) was used to determine SFBs' cell viability. About $6 \times 10^{4}$ cells $/ \mathrm{ml}$ of SFBs in the logarithmic phase were sowed into the wells of 96-well plates, and then maintained in a $5 \% \mathrm{CO}_{2}$ atmosphere at $37^{\circ} \mathrm{C}$ for $12 \mathrm{~h}$. Afterwards, SFBs were handled as described above. Cells were then maintained for 12,24 , and $48 \mathrm{~h}$ respectively. $10 \mu \mathrm{l}$ of CCK reagent was then added into the wells. After that, cells were maintained for $3 \mathrm{~h}$. The absorbance at $450 \mathrm{~nm}$ was read by a Microplate reader (Bio-Rad Laboratories, Inc., Hercules, CA, USA). Cell viability was evaluated by the percentage of cell survival.

Western blot analysis. A total of $12 \%$ sodium dodecyl sulfate polyacrylamide gel electrophoresis (SDS-PAGE) was used to segregate proteins lysates of cultured SFBs, which were then transferred to a PVDF membrane (EMD Millipore, Billerica, MA, USA). Blotting was carried out by using specific antibodies (anti-Dvl-2, dilution, 1:1,000, cat. no. ab22616, rabbit anti-human; anti-OPG, dilution, 1:1,000, cat. no. ab73400, rabbit anti-human; anti-RANKL, dilution, 1:1,000, cat. no. ab9957, rabbit anti-human; anti-ALP, dilution, 1:1,000, cat. no. ab83259, rabbit anti-human; anti-ON, dilution, 1:1,000, cat. no. ab8448, rabbit anti-human; anti-osteocalcin (OCN), dilution, 1:500, cat. no. ab93876, rabbit anti-human; anti-osterix, dilution, 1:1,000, cat. no. ab22552, rabbit anti-human; anti-Wnt3a, dilution, 1:1,000, cat. no. ab28472, rabbit anti-human; anti- $\beta$-catenin, dilution, 1:5,000, cat. no. ab32572, rabbit anti-human; anti-Runx-2, dilution, 1:1,000, cat. no. ab23981, rabbit anti-human; anti- $\beta$-actin, dilution, 1:2,000, cat. no. ab8227, rabbit anti-human; all from Abcam, Cambridge, UK). Horseradish peroxidase-conjugated secondary antibodies (dilution, 1:5,000, cat. no. ab205718, goat anti-rabbit; Abcam) were supplemented and incubated for $1 \mathrm{~h}$ at room temperature. Enhanced chemiluminescent reagents (EMD Millipore) using an ECL system (Amersham; GE Healthcare, Chicago, IL, USA) were performed on the evaluation of results.

Reverse transcription-quantitative reverse transcription $P C R$ (RT-qPCR) analysis. Total RNA was extracted from cultured SFBs by TRIzol reagent (Beijing Solarbio Science \& Technology Co., Ltd., Beijing, China). RNA was reverse transcribed to cDNA by Reverse Transcription kit (Beijing Solarbio Science \& Technology Co., Ltd.) according to the direction. RT-qPCR analysis was performed on ABI 7500 Thermocycler (Applied Biosystems Thermo Fisher Scientific, Inc.). PCR cycling conditions were as follows: One pretreatment at $95^{\circ} \mathrm{C}$ for $10 \mathrm{~min}, 94^{\circ} \mathrm{C}$ for $15 \mathrm{sec}, 62^{\circ} \mathrm{C}$ for $45 \mathrm{sec}\left(45\right.$ cycles), $94^{\circ} \mathrm{C}$ for $15 \mathrm{sec}, 62^{\circ} \mathrm{C}$ for $1 \mathrm{~min}, 95^{\circ} \mathrm{C}$ for $15 \mathrm{sec}$, a final extension at $75^{\circ} \mathrm{C}$ for $10 \mathrm{~min}$ and was held at $4^{\circ} \mathrm{C}$. Relative expressions of target genes were calculated by $2^{-\Delta \Delta \mathrm{CT}}$ method (27). The primers were purchased from Shanghai Invitrogen Biotechnology Co., Ltd. (Shanghai, China): Dvl-2 forward, 5'-CATCCAGCCAAT TGACCCTG-3' and reverse, 5'-AGGGATGGTGATCTTGA GCC-3' (product, 241 bp); OPG forward, 5'-GGCACCAAA GTAAACGCAGA-3' and reverse, 5'-TCCCGGTAAGCTTTC CATCA-3' (product, 228 bp); RANKL forward, 5'-CGCTCG TGTTTCTGGACATC-3' and reverse, 5'-GGGGCTGCAGTA TAGACACT-3' (product, 233 bp); ALP forward, 5'-CCTCTT CCCCTTCCTGGTG-3' and reverse, 5'-GATGCCACAAGT GTCAGGAC-3' (product, 196 bp); ON forward, 5'-CAACGA AAGCCATGACCACA-3' and reverse, 5'-ACCTCGGCCATC ATATGTGT-3' (product, 247 bp); OCN forward, 5'-GCAGAG TCCAGCAAAGGTG-3' and reverse, 5'-TCACAGTCCGGA TTGAGCTC-3' (product, $161 \mathrm{bp}$ ); osterix forward, 5'-TCT CTGGACATGACACACCC-3' and reverse, 5'-AGGGGA GCAAAGTCAGATGG-3' (product, 233 bp); Wnt3a forward, 5'-ATCGAGTTTGGTGGGATGGT-3' and reverse, 5'-CGC 
A

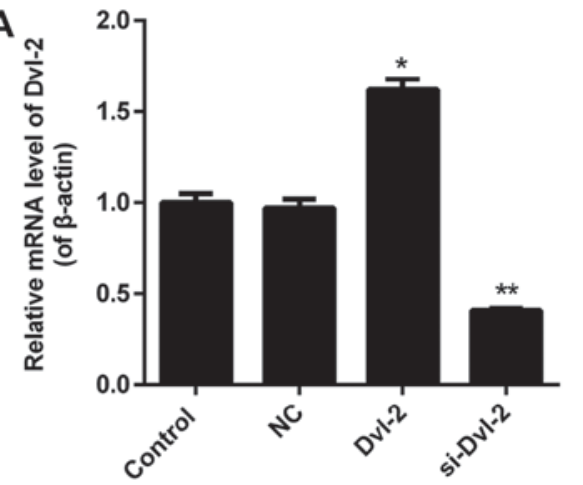

B

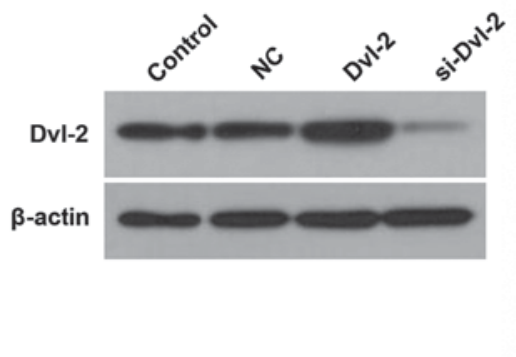

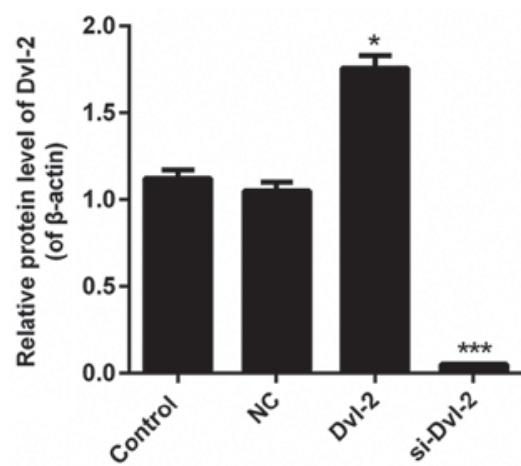

Figure 1. Over-expression and interference of Dvl-2 in SFBs. (A) Reverse transcription-quantitative polymerase chain reaction and (B) western blot analysis were performed to detect the mRNA and protein expression levels of Dvl-2 in SFBs, SFBs transfected with empty vector, SFBs transfected with Dvl-2 and SFBs transfected with si-Dvl-2. ${ }^{*} \mathrm{P}<0.05,{ }^{* *} \mathrm{P}<0.01$ and ${ }^{* * *} \mathrm{P}<0.001$ vs. NC. Dvl-2, dishevelled-2; SFB, synovial fibroblasts; si, short interfering; NC, negative control.

TGTCGTACTTGTCCTTG-3' (product, 238 bp); $\beta$-catenin forward, 5'-AGTTCCTTACCGTCCCCAAG-3' and reverse, 5'-CAGACACGCCTGTTTCGAAT-3' (product, 249 bp); Runx-2 forward, 5'-ATTCTGCTGAGCTCCGGAAT-3' and reverse, 5'-AGCTTCTGTCTGTGCCTTCT-3' (product, $211 \mathrm{bp}$ ); and $\beta$-actin forward, 5'-GTTACAGGAAGTCCCTCA CCC-3' and reverse, 5'-CAGACCTGGGCCATTCAGAAA-3' (product, 194 bp). $\beta$-actin was used as the control of the input RNA level.

Statistical analysis. Results are shown as the mean \pm SD. Following Dunnet's test, all experimental data were analyzed by one-way analysis of variance followed by Dunnett's multiple comparisons post hoc test. GraphPad Prism version 6.0 (GraphPad Software, Inc., La Jolla, CA, USA) was used to perform the statistical analysis. The statistical significance was defined as $\mathrm{P}<0.05$.

\section{Results}

Over-expression and interference of Dvl-2 in SFBs. RNA and siRNA vectors targeting Dvl-2 gene named Dvl-2 and si-Dvl-2 were constructed in our study. After being transfecting with Dvl-2, the expression level of Dvl-2 in SFBs was clearly upregulated, and the knockdown efficiency was about $60 \%$ in SFBs after being stably transfected with si-Dvl-2 $(\mathrm{P}<0.05$; Fig. 1A and B).

Dvl-2 silence reduced the cell viability of SFBs. CCK-8 assay was carried out to determine the cell viability of SFBs coped with the treatment groups as previously described. The results shown that both the over-expression of Dvl-2 over-expression and depletion of Dvl-2 slightly reduced the cell viability of SFBs (Fig. 2). No significant difference was found among these groups.

Dvl-2 affected the activity of ALP in SFBs. We also evaluated the activity of ALP in SFBs coped with the treatment groups as described above. An obvious increase of ALP activity was observed in Dvl-2 group compared with $\mathrm{NC}(\mathrm{P}<0.05$; Fig. 3A). However, the ALP activity in SFBs was markedly reduced by si-Dvl-2 ( $\mathrm{P}<0.01$; Fig. 3A). Therefore, it was determined that Dvl-2 silence lessened the activity of ALP in SFBs.

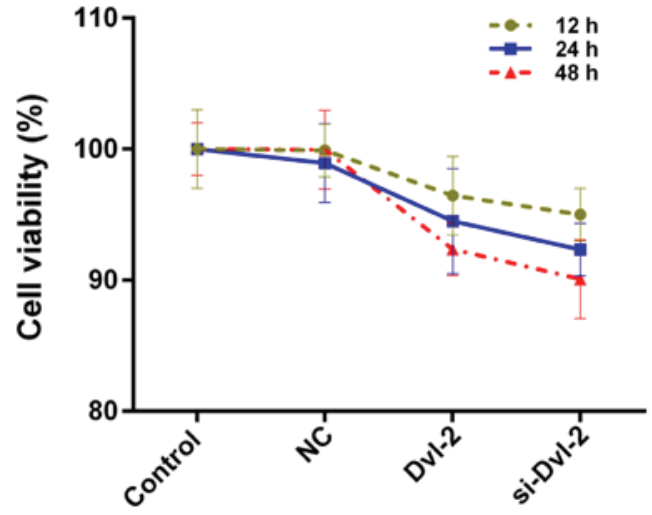

Figure 2. Effect of Dvl-2 on the cell viability of SFBs. A Cell Counting kit- 8 assay was performed to measure the cell viability of SFBs, SFBs transfected with empty vector, SFBs transfected with Dvl-2 and SFBs transfected with si-Dvl-2 at 12, 24 and 48 h. Dvl-2, dishevelled-2; SFB, synovial fibroblasts; si, short interfering; NC, negative control.

Dvl-2 regulated the osteogenic differentiation in SFBs. To further investigate the funtion of Dvl-2 in the osteogenic differentiation of SFBs, we assessed the expression of osteogenic factors in SFBs that were transfected with Dvl-2 or si-Dvl-2, using OPG, RANKL, ALP, ON, OCN, and osterix. We found that a rise in the OPG/RANKL ratio in Dvl-2 group, which is in contrast to a decline in the si-Dvl-2 group $(\mathrm{P}<0.05$; Fig. 3B and C). Moreover, compared to control group, the expression levels of $\mathrm{ON}, \mathrm{OCN}$, and osterix in SFBs transfected with si-Dvl-2 were significantly upregulated However, a sharp decrease of ALP expression was observed in si-Dvl-2 group; and an increase in Dvl-2 group $(\mathrm{P}<0.05$; Fig. $4 \mathrm{~A}$ and $\mathrm{B})$.

Dvl-2 affected the activity of Runx-2. For the purpose of exploring the mechanisms of Dvl-2 in the osteogenic differentiation of SFBs, we therefore measured the expression levels of Runx-2 in SFBs from all treatment groups. The results indicate that compared to control group, the expression levels of Runx-2 was upregulated by Dvl-2 and downregulated by si-Dvl2. Moreover, though the expression of Wnt3a and $\beta$-catenin increased at the presence of Dvl-2, the depletion of Dvl-2 decreased the levels of Wnt $3 a$ and $\beta$-catenin $(\mathrm{P}<0.05$; Fig. 5A and B). Furthermore, Wnt3a was inhibited to estimate the effect of Dvl-2. The expression of Wnt3a was shown in 

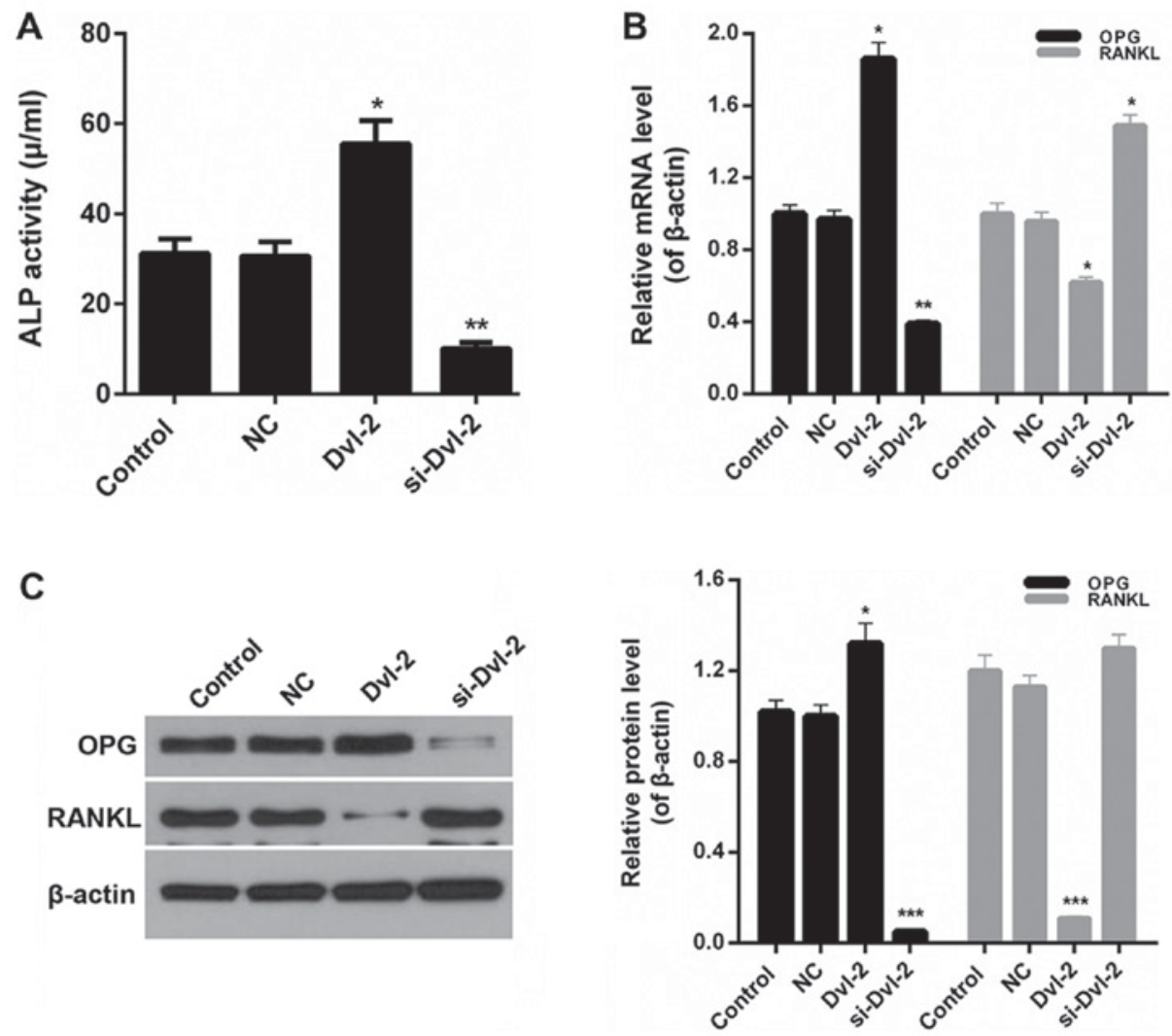

Figure 3. Effect of Dvl-2 on ALP activity and OPG and RANKL expression in SFBs. SFBs were transfected with empty vector, Dvl-2 and si-Dvl-2. (A) An ALP test kit was used to measure the activity of ALP in SFBs. (B) Reverse transcription-quantitative polymerase chain reaction (C) and western blot analysis were performed to evaluate the mRNA and protein expression levels of OPG and RANKL in SFBs. ${ }^{*} \mathrm{P}<0.05,{ }^{* *} \mathrm{P}<0.01$ and ${ }^{* * * *} \mathrm{P}<0.001$ vs. NC. Dvl-2, dishevelled-2; SFB, synovial fibroblasts; si, short interfering; ALP, alkaline phosphatase; OPG, osteoprotegerin; RANKL, receptor activator of nuclear factor- $\mathrm{kB}$ ligand; NC, negative control.

A
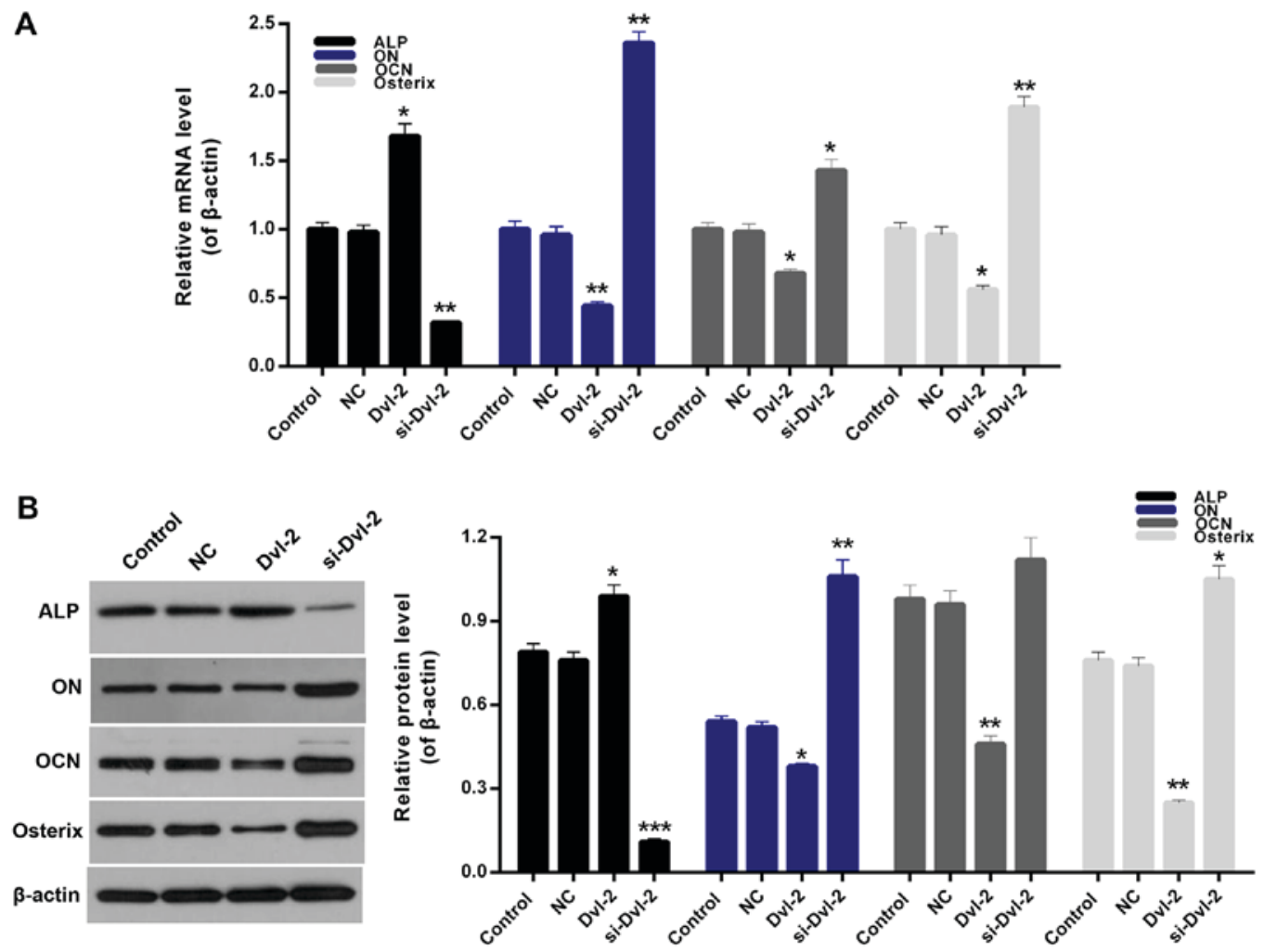

Figure 4. Effect of Dvl-2 on the osteogenic differentiation of SFBs. (A) Reverse transcription-quantitative polymerase chain reaction and (B) western blot analysis were performed to determine the mRNA and protein expression levels of ALP, ON, OCN and osterix in SFBs, SFBs transfected with empty vector, SFBs transfected with Dvl-2 and SFBs transfected with si-Dvl-2. ${ }^{*} \mathrm{P}<0.05,{ }^{* *} \mathrm{P}<0.01$ and ${ }^{* * * *} \mathrm{P}<0.001$ vs. NC. Dvl-2, dishevelled-2; SFB, synovial fibroblasts; si, short interfering; ALP, alkaline phosphatase; $\mathrm{ON}$, osteonectin; $\mathrm{OCN}$, osteocalcin; $\mathrm{NC}$, negative control. 

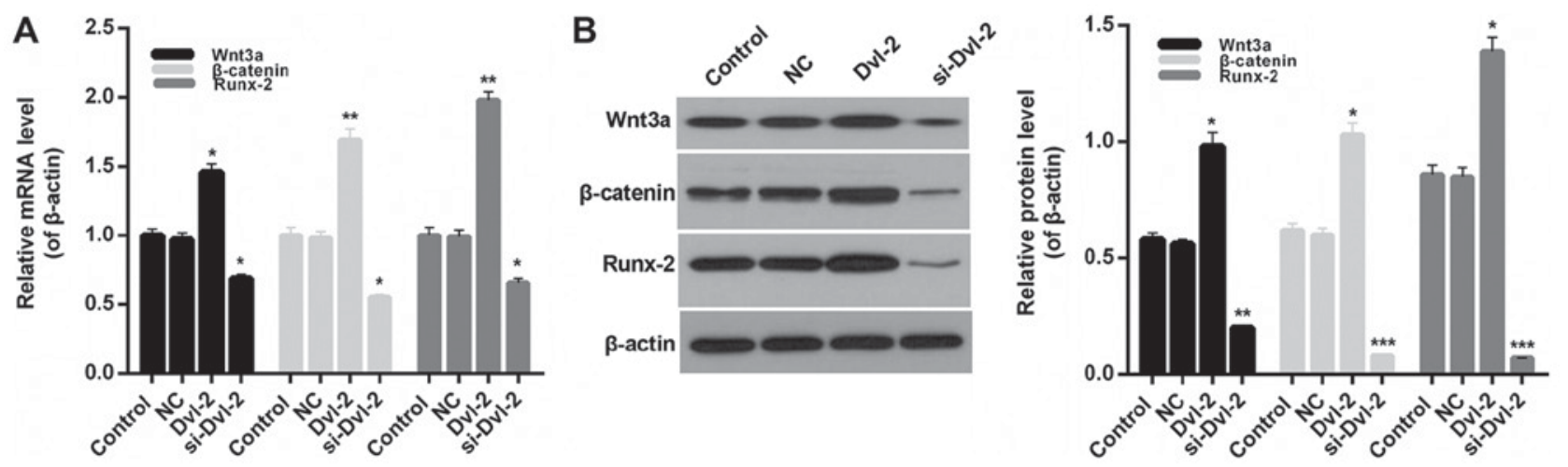

Figure 5. Effect of Dvl-2 on the expression of Runx-2, Wnt3a and $\beta$-catenin. (A) Reverse transcription-quantitative polymerase chain reaction and (B) western blot analysis were performed to determine the mRNA and protein expression levels of Wnt3a, $\beta$-catenin and Runx-2 in SFBs, SFBs transfected with empty vector, SFBs transfected with Dvl-2 and SFBs transfected with si-Dvl-2. ${ }^{*} \mathrm{P}<0.05,{ }^{* *} \mathrm{P}<0.01$ and ${ }^{* * *} \mathrm{P}<0.001$ vs. NC. Dvl-2, dishevelled-2; SFB, synovial fibroblasts; si, short interfering; Runx-2, runt-related transcription factor 2; NC, negative control.
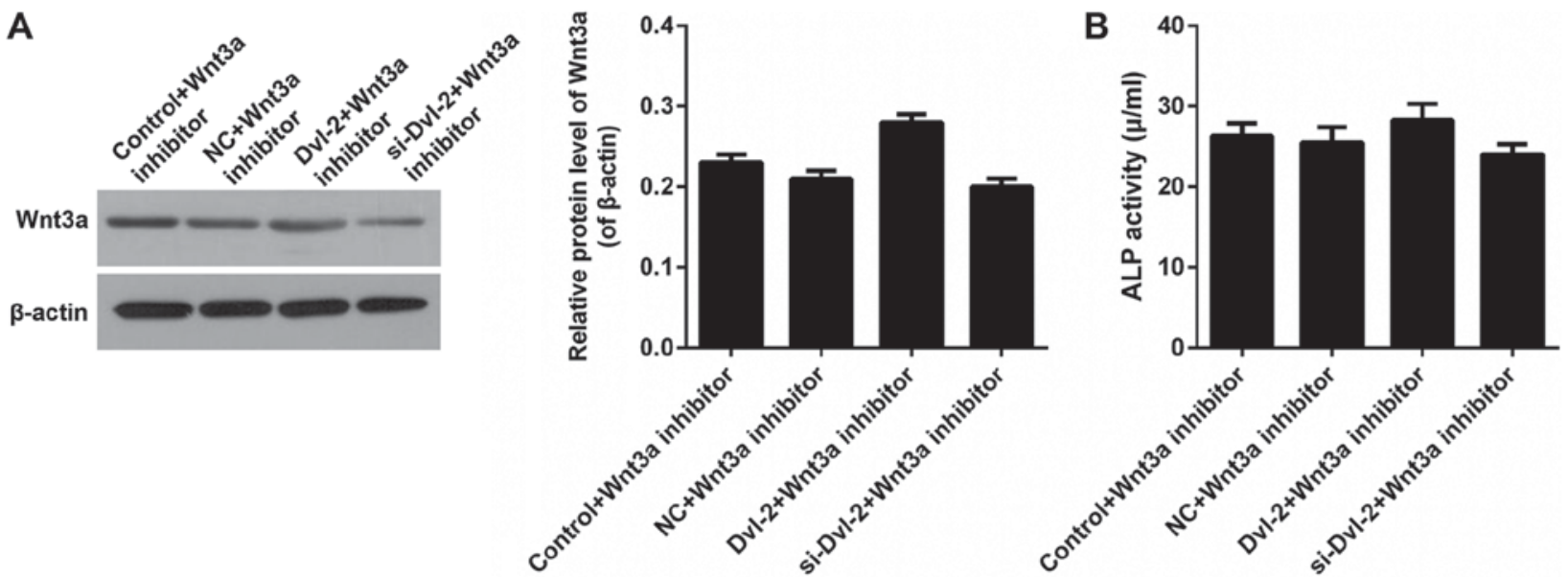

Figure 6. Wnt3a impacted the activity of ALP in SFBs. SFBs, SFBs transfected with empty vector, SFBs transfected with Dvl-2 and SFBs transfected with si-Dvl-2 were treated with Wnt3a inhibitor. (A) A western blot assay was performed to determine the protein expression levels of Wnt3a in SFBs. (B) An ALP test kit was used to measure the activity of ALP in SFBs. Dvl-2, dishevelled-2; SFB, synovial fibroblasts; si, short interfering; NC, negative control.

Fig. 6A. After the inhibition of Wnt signaling by IWR-1-endo, there was no significant difference of ALP activity in SFBs from all of the treatment groups (Fig. 6B).

\section{Discussion}

$\mathrm{OA}$ is one of the articular degenerative disorders. It can lead to bone destruction around the joint, ossification in the surrounding tissues, subsequent loss of bony rigidity and even joint activity (28). Based on the pathological investigation of OA ossification sites, it was considered that non-osteocytes at the attachment sites can proliferate and gradually differentiates into chondrocytes. The fiber textures at attachment sites gradually grow thicker and the cartilage-based pathological nodules will occur as a consequence (29). Furthermore, these possessing secretion function cells releases a large number of ALP matrix vesicles, which will cause the partial formation of hydeoxyapatite crystal and then gradually calcification (30-32). Multiple cell types are involved in the progress of osteogenic differentiation. SFBs have been widely investigated in the previous research about OA (33-36). And SFB palyed important roles both in bone resorption and bone formation $(37,38)$. Thus, in this study, we selected SFBs as our research objects to further explore the the osteogenic differentiation in OA.

Previous studies showed that the close connections between Dvl-2 and Wnt pathway (39-41). However, to the best of our knowledge, the roles and mechanisms of Dvl-2 in the osteogenic differentiation of SFBs have not been studied yet. Thus, we selected Dvl-2 as the study object, and transfected the SFBs with Dvl-2 and si-Dvl-2. Over-expression and silencing of Dvl-2 in SFBs were observed, and the knockdown efficiency of Dvl-2 was about $60 \%$. We first measured the cell viability of SFBs, which are transfected with Dvl-2 and si-Dvl-2. The results indicated that Dvl-2 silence could inhibit the cell viability of SFBs, especially for $48 \mathrm{~h}$-treatment. Then, we measured the activity of ALP in SFBs, which were transfected with Dvl-2 and si-Dvl-2. The results showed that Dvl-2 significantly influenced the ALP activity in SFBs. In order to investigate the functions of Dvl-2 in the osteogenic differentiation of SFBs, the expressions of OPG and RANKL in SFBs were examined in reference to previous studies $(6,7)$. Based on the experimental results, we found that the OPG/RANKL ratio was remarkably reducced by si-Dvl-2. 
Moreover, over-expression of Dvl-2 significantly enhanced the OPG/RANKL ratio in SFBs. Additionally, Dvl-2 silence significantly reduced the ALP expression, while it upregulated the expression levels of ON, OCN, and osterix in SFBs. It was confirmed that Dvl-2 suppressed bone absorption of SFBs in $\mathrm{OA}$ by regulating the expression levels of OPG, RANKL, ALP, ON, OCN, and osterix.

In different stages of osteogenic differentiation, signaling pathways involved/participated are not the same. It has been proved that ossification was largely affected by BMP pathway at an early stage, while the Wnt pathway impacts ossification in the advanced ossification (42-44). Among them, Wnt/ $\beta$-catenin pathway plays an important role in stem cell differentiation, bone formation, and the regulation of balance from the embryonic period (45). The abnormal regulation of Wnt pathway is closely associated with the bony ankylosis in OA, and was considered as one of the important factors in osteogenic differentiation. In the present study, the Wnt3a, $\beta$-catenin, and Runx-2 expressions in SFBs were studied. Our results showed that Dvl-2 silence significantly downregulated the expression levels of Wnt3a, $\beta$-catenin, and Runx-2 in SFBs. After downregulating the Wnt3a expression, we found that there was no significant difference in the activities of ALP in SFBs. Such results remind us of that Dvl-2 regulated the activity of Runx-2 by affecting the Wnt pathway in SFBs. Thus, it can concluded that Dvl-2 modulated the osteogenic differentiation of SFBs in OA via Wnt/ $\beta$-catenin/Runx-2 pathway, to some extent.

Taken together, our research demonstrated that the Dvl-2 plays a critical role in osteogenic differentiation of SFBs in OA, which was related to the Wnt pathway. Also, the results provided a new thread for understanding the pathogenesis of $\mathrm{OA}$ and put forward a fascinating approach for the therapy of OA.

In conclusoin, our study highlights that Dvl-2 silence modulates the ossification of SFBs in OA by downregulating the Wnt pathway. The findings of our research are crucial to unfolding the mechanisms of Dvl-2 in the ossification of SFBs. The potential effects of Dvl-2 in the ossification of SFBs suggest that Dvl-2 might be an effective target for OA therapies.

\section{Acknowledgements}

Not applicable.

\section{Funding}

No funding was received.

\section{Availability of data and materials}

All data generated or analyzed during this study are included in this published article.

\section{Authors' contributions}

LZ wrote the main manuscript. LL performed the experiments. YM designed the study. LZ and LL performed data analysis. LZ, LL and YM contributed to manuscript revisions and all authors reviewed the final version of the manuscript.

\section{Ethics approval and consent to participate}

The present study was approved by the Ethics Committee of Jining No. 1 People's Hospital. Written informed consent was obtained from all patients prior to their inclusion within the study.

\section{Consent for publication}

Written informed consent was obtained from all patients for the publication of their data.

\section{Competing interests}

The authors declare that they have no competing interests.

\section{References}

1. Glyn-Jones S, Palmer AJ, Agricola R, Price AJ, Vincent TL, Weinans H and Carr AJ: Osteoarthritis. Lancet 386: 376-387, 2015.

2. Johnson VL and Hunter DJ: The epidemiology of osteoarthritis. Best Pract Res Clin Rheumatol 28: 5-15, 2014.

3. Benito MJ, Veale DJ, FitzGerald O, van den Berg WB and Bresnihan B: Synovial tissue inflammation in early and late osteoarthritis. Ann Rheum Dis 64: 1263-1267, 2005.

4. Lambert C, Dubuc JE, Montell E, Vergés J, Munaut C, Noë A and Henrotin Y: Gene expression pattern of cells from inflamed and normal areas of osteoarthritis synovial membrane. Arthritis Rheumatol 66: 960-968, 2014.

5. Iwanaga T, Shikichi M, Kitamura H, Yanase $H$ and NozawaInoue $\mathrm{K}$ : Morphology and functional roles of synoviocytes in the joint. Arch Histol Cytol 63: 17-31, 2000.

6. Danks L, Komatsu N, Guerrini MM, Sawa S, Armaka M, Kollias G, Nakashima T and Takayanagi H: RANKL expressed on synovial fibroblasts is primarily responsible for bone erosions during joint inflammation. Ann Rheum Dis 75: 1187-1195, 2016.

7. Miyashita T, Kawakami A, Nakashima T, Yamasaki S, Tamai M, Tanaka F, Kamachi M, Ida H, Migita K, Origuchi T, et al: Osteoprotegerin (OPG) acts as an endogenous decoy receptor in tumour necrosis factor-related apoptosis-inducing ligand (TRAIL)-mediated apoptosis of fibroblast-like synovial cells. Clin Exp Immunol 137: 430-436, 2004.

8. Sharma U, Pal D and Prasad R: Alkaline phosphatase: An overview. Indian J Clin Biochem 29: 269-278, 2014.

9. Delgado-Calle J, Sanudo C, Sánchez-Verde L, Garcia-Renedo RJ, Arozamena J and Riancho JA: Epigenetic regulation of alkaline phosphatase in human cells of the osteoblastic lineage. Bone 49: 830-838, 2011.

10. Ek-Rylander B and Andersson G: Osteoclast migration on phosphorylated osteopontin is regulated by endogenous tartrate-resistant acid phosphatase. Exp Cell Res 316: 443-451, 2010.

11. Prins HJ, Braat AK, Gawlitta D, Dhert WJ, Egan DA, TijssenSlump E, Yuan H, Coffer PJ, Rozemuller H and Martens AC: In vitro induction of alkaline phosphatase levels predicts in vivo bone forming capacity of human bone marrow stromal cells. Stem Cell Res 12: 428-440, 2014.

12. Seo HJ, Cho YE, Kim T, Shin HI and Kwun IS: Zinc may increase bone formation through stimulating cell proliferation, alkaline phosphatase activity and collagen synthesis in osteoblastic MC3T3-E1 cells. Nutr Res Pract 4: 356-361, 2010.

13. Sardiwal S, Magnusson P, Goldsmith DJ and Lamb EJ: Bone alkaline phosphatase in CKD-mineral bone disorder. Am J Kidney Dis 62: 810-822, 2013.

14. Gulseren G, Yasa IC, Ustahuseyin O, Tekin ED, Tekinay AB and Guler MO: Alkaline phosphatase-mimicking peptide nanofibers for osteogenic differentiation. Biomacromolecules 16: 2198-2208, 2015.

15. Chien AJ, Conrad WH and Moon RT: A Wnt survival guide: From flies to human disease. J Invest Dermatol 129: 1614-1627, 2009.

16. James RG, Conrad WH and Moon RT: Beta-catenin-independent Wnt pathways: Signals, core proteins and effectors. Methods Mol Biol 468: 131-144, 2008. 
17. Baron R and Kneissel M: WNT signaling in bone homeostasis and disease: From human mutations to treatments. Nat Med 19: 179-192, 2013.

18. Sen M: Wnt signalling in rheumatoid arthritis. Rheumatology (Oxford) 44: 708-713, 2005.

19. Gao C and Chen YG: Dishevelled: The hub of Wnt signaling. Cell Signal 22: 717-727, 2010.

20. Blom AB, van Lent PL, van der Kraan PM and van den Berg WB: To seek shelter from the WNT in osteoarthritis? WNT-signaling as a target for osteoarthritis therapy. Curr Drug Targets 11: 620-629, 2010

21. Abraham DJ, Shiwen X, Black CM, Sa S, Xu Y and Leask A: Tumor necrosis factor alpha suppresses the induction of connective tissue growth factor by transforming growth factor-beta in normal and scleroderma fibroblasts. J Biol Chem 275 : $15220-15225,2000$

22. Wallingford JB and Habas R: The developmental biology of Dishevelled: An enigmatic protein governing cell fate and cell polarity. Development 132: 4421-4436, 2005.

23. Pulvirenti T, Van Der Heijden M, Droms LA, Huse JT, Tabar V and Hall A: Dishevelled 2 signaling promotes self-renewal and tumorigenicity in human gliomas. Cancer Res 71: 7280-7290, 2011.

24. Yang Y, Jiao L, Hou J, Xu C, Wang L, Yu Y, Li Y, Yang C, Wang X and Sun Y: Dishevelled-2 silencing reduces androgen-dependent prostate tumor cell proliferation and migration and expression of Wnt-3a and matrix metalloproteinases. Mol Biol Rep 40: 4241-4250, 2013

25. Zhou G, Ye J, Sun L, Zhang Z and Feng J: Overexpression of Dishevelled-2 contributes to proliferation and migration of human esophageal squamous cell carcinoma. J Mol Histol 47: 287-295, 2016.

26. Sadouk MB, Pelletier JP, Tardif G, Kiansa K, Cloutier JM and Martel-Pelletier J: Human synovial fibroblasts coexpress IL-1 receptor type I and type II mRNA. The increased level of the IL-1 receptor in osteoarthritic cells is related to an increased level of the type I receptor. Lab Invest 73: 347-355, 1995.

27. Arocho A, Chen B, Ladanyi M and Pan Q: Validation of the 2-DeltaDeltaCt calculation as an alternate method of data analysis for quantitative PCR of BCR-ABL P210 transcripts. Diagn Mol Pathol 15: 56-61, 2006.

28. Loeser RF, Goldring SR, Scanzello CR and Goldring MB Osteoarthritis: A disease of the joint as an organ. Arthritis Rheum 64: 1697-1707, 2012.

29. Grenier S, Bhargava MM and Torzilli PA: An in vitro model for the pathological degradation of articular cartilage in osteoarthritis. J Biomech 47: 645-652, 2014.

30. Anderson HC, Sipe JB, Hessle L, Dhanyamraju R, Atti E, Camacho NP, Millán JL and Dhamyamraju R: Impaired calcification around matrix vesicles of growth plate and bone in alkaline phosphatase-deficient mice. Am J Pathol 164: 841-847, 2004.

31. Fallon MD, Whyte MP and Teitelbaum SL: Stereospecific inhibition of alkaline phosphatase by L-tetramisole prevents in vitro cartilage calcification. Lab Invest 43: 489-494, 1980.

32. Yadav MC, Simão AM, Narisawa S, Huesa C, McKee MD, Farquharson C and Millán JL: Loss of skeletal mineralization by the simultaneous ablation of PHOSPHO1 and alkaline phosphatase function: A unified model of the mechanisms of initiation of skeletal calcification. J Bone Miner Res 26: 286-297, 2011.
33. Carrión M, Juarranz Y, Pérez-Garcia S, Jimeno R, Pablos JL, Gomariz RP and Gutiérrez-Cañas I: RNA sensors in human osteoarthritis and rheumatoid arthritis synovial fibroblasts: Immune regulation by vasoactive intestinal peptide. Arthritis Rheum 63: 1626-1636, 2011.

34. Eisinger K, Bauer S, Schäffler A, Walter R, Neumann E, Buechler C, Müller-Ladner U and Frommer KW: Chemerin induces CCL2 and TLR4 in synovial fibroblasts of patients with rheumatoid arthritis and osteoarthritis. Exp Mol Pathol 92: 90-96, 2012.

35. Haas S and Straub RH: Disruption of rhythms of molecular clocks in primary synovial fibroblasts of patients with osteoarthritis and rheumatoid arthritis, role of IL-1beta/TNF. Arthritis Res Ther 14: R122, 2012.

36. Qin Y, Chen Y, Wang W, Wang Z, Tang G, Zhang P, He Z, Liu Y, Dai SM and Shen Q: HMGB1-LPS complex promotes transformation of osteoarthritis synovial fibroblasts to a rheumatoid arthritis synovial fibroblast-like phenotype. Cell Death Dis 5: e1077, 2014

37. Galicka A, Surazynski A, Wołczyński S, Palka J, Popko J and Gindzieński A: Phenotype variability in a daughter and father with mild osteogenesis imperfecta correlated with collagen and prolidase levels in cultured skin fibroblasts. Ann Clin Biochem 42: 80-84, 2005.

38. Bhattaram $P$ and Chandrasekharan U: The joint synovium: A critical determinant of articular cartilage fate in inflammatory joint diseases. Semin Cell Dev Biol 62: 86-93, 2017.

39. Gonzalez-Sancho JM, Greer YE, Abrahams CL, Takigawa Y, Baljinnyam B, Lee KH, Lee KS, Rubin JS and Brown AM: Functional consequences of Wnt-induced dishevelled 2 phosphorylation in canonical and noncanonical Wnt signaling. J Biol Chem 288: 9428-9437, 2013.

40. Smalley MJ, Signoret N, Robertson D, Tilley A, Hann A, Ewan K, Ding Y, Paterson H and Dale TC: Dishevelled (Dvl-2) activates canonical Wnt signalling in the absence of cytoplasmic puncta. J Cell Sci 118: 5279-5289, 2005.

41. Yokoyama N and Malbon CC: Dishevelled-2 docks and activates Src in a Wnt-dependent manner. J Cell Sci 122: 4439-4451, 2009.

42. Kamiya N, Ye L, Kobayashi T, Mochida Y, Yamauchi M, Kronenberg HM, Feng JQ and Mishina Y: BMP signaling negatively regulates bone mass through sclerostin by inhibiting the canonical Wnt pathway. Development 135: 3801-3811, 2008.

43. Kawaguchi H: Regulation of osteoarthritis development by Wnt-beta-catenin signaling through the endochondral ossification process. J Bone Miner Res 24: 8-11, 2009.

44. Yu YY, Lieu S, Lu C and Colnot C: Bone morphogenetic protein 2 stimulates endochondral ossification by regulating periosteal cell fate during bone repair. Bone 47: 65-73, 2010.

45. Tamamura Y, Otani T, Kanatani N, Koyama E, Kitagaki J, Komori T, Yamada Y, Costantini F, Wakisaka S, Pacifici M, et al: Developmental regulation of Wnt/beta-catenin signals is required for growth plate assembly, cartilage integrity, and endochondral ossification. J Biol Chem 280: 19185-19195, 2005.

This work is licensed under a Creative Commons Attribution-NonCommercial-NoDerivatives 4.0 International (CC BY-NC-ND 4.0) License. 